\title{
Minecoside promotes apoptotic progression through STAT3 inactivation in breast cancer cells
}

\author{
BUYUN KIM ${ }^{1,2}$, KI YONG LEE ${ }^{3}$ and BYOUNGDUCK PARK ${ }^{1}$ \\ ${ }^{1}$ College of Pharmacy, Keimyung University, Dalseo-Gu, Daegu, North Gyeongsang 704-701; \\ ${ }^{2}$ Korean Medicine Application Center, Korea Institute of Oriental Medicine, Daegu, North Gyeongsang 701-300; \\ ${ }^{3}$ College of Pharmacy, Korea University, Sejong 339-770, Republic of Korea
}

Received August 24, 2021; Accepted January 4, 2022

DOI: $10.3892 / 01.2022 .13214$

\begin{abstract}
Breast cancer is one of the most common malignant tumors in women worldwide, and is a major cause of mortality and morbidity in cancer patients. Constitutive activation of STAT3 has been found in a variety of malignant tumors, including breast cancer. Since STAT3 activation is capable of regulating various important features of tumor cells, identification of a novel STAT3 inhibitor is considered a potential strategy for treating breast cancer. The aim of the present study was to examine whether minecoside (MIN), an active compound extracted from Veronica peregrina L., exerts an antitumor effect by inhibiting STAT3 signaling pathway in MDA-MB-231 cells. The results revealed that MIN inhibited the constitutive STAT3 activation in a dose- and time-dependent manner. MIN also blocked the nuclear translocation of STAT3 and suppressed STAT3-DNA binding. In addition, MIN downregulated the STAT3-mediated expression of proteins such as Bcl-xL, Bcl-2, CXCR4, VEGF, and cyclin D1. Subsequently, MIN promoted the caspase-dependent apoptosis in MDA-MB-231 cells. Overall, results of the present study provide evidence that MIN exerted anticancer activity via inhibition of the STAT3 signaling pathway. Further studies using animal models are required to determine the potential of this molecule as an anticancer drug.
\end{abstract}

\section{Introduction}

Breast cancer is one of the most common malignant tumors in women worldwide, and triple-negative breast cancer accounts for $15-20 \%$ of all breast cancer cases $(1,2)$. At present, various

Correspondence to: Dr Byoungduck Park, College of Pharmacy, Keimyung University, 1095 Dalgubeoldaero, Dalseo-Gu, Daegu, North Gyeongsang 704-701, Republic of Korea

E-mail: bdpark@kmu.ac.kr

Dr Ki Yong Lee, College of Pharmacy, Korea University, Sejong Campus 2511 Sejong-ro, Sejong 339-770, Republic of Korea

E-mail: kylee11@korea.ac.kr

Key words: breast cancer, minecoside, STAT3 inactivation clinical trials have been conducted to treat and delay the progression of breast cancer, but mortality and morbidity among patients remain high (3). Several carcinogens and signaling pathways are known to be involved in the progression of breast cancer. Among them, signal transducer and activator of transcription 3 (STAT3) has been reported to play a pivotal role in breast cancer development (4). Unlike normal cells, in which the activity of STAT3 is strictly regulated, STAT3 activity in breast cancer contributes to tumorigenesis in a multifaceted manner (5). Interleukin-6 (IL-6), G-protein-coupled receptors (GPCRs), and Toll-like receptors (TLRs) are known to be involved in STAT3 activation in various types of cancer, including colon cancer $(6,7)$. Once STAT3 signaling is activated, it induces carcinogenesis through the expression of various genes associated with apoptosis (survivin, Bcl-xL, and Bcl-2), proliferation (cyclin D1), and angiogenesis (VEGF) $(8,9)$. Additionally, several protein tyrosine phosphatases (PTPs) promote the death of cancer cells by STAT3 inactivation $(10,11)$. SH2 domain containing phosphatase 1/2 (SHP-1/2) and protein tyrosine phosphatase 1B (PTP-1B) are known to be associated with STAT3 inactivation . SHP-1 is highly expressed in normal lymphocytes, whereas its expression is decreased in most cancer cells (12). In addition, SHP-1 expression in cancer cells effectively suppresses target genes such as VEGF-1, cyclin D1, and survivin (13). Thus, dephosphorylation of STAT3 by increasing the expression of SHP-1 may be an efficient strategy for the treatment of various cancer types.

In previous decades, many researchers have suggested that natural compounds may act as potent anti-cancer drugs with high efficacy and low side effects. Various candidates have been studied and examined for their anti-cancer properties and underlying mechanisms. Minecoside (MIN), an active compound extracted from Veronica peregrina L., belongs to the family Scrophulariaceae (14). The entire plant has been used as a traditional drug for the treatment of menstrual irregularities, fractures, and traumatic injuries (14). Moreover, several compounds from Veronica peregrina L. have been reported to exhibit antioxidant activity (14). A recent study revealed that MIN suppressed the invasive capability of cancer cells by inhibiting CXCR4 expression via blocking NF- $\kappa B$ (15). However, the mechanism underlying the regulation of STAT3 activation by MIN has not been completely 
understood. Therefore, we investigated whether MIN could modulate the apoptosis of breast cancer cells by regulating the STAT3 signaling pathway.

\section{Materials and methods}

Purity analysis of minecoside. Minecoside was isolated from the Catalpa ovata according to a previously protocol (16). Purity analysis of isolated minecoside was carried on Shiseido CapCell PAK C18 column (Sigma-Aldrich) particle size $5 \mu \mathrm{m}$ (150x4.6 mm) using a Waters 2695 system (Waters Corporation). The mobile phase consisted of water with $0.1 \%$ formic acid (solvent A), acetonitrile with $0.1 \%$ formic acid (solvent B), which were applied in the following gradient elution: $5 \% \mathrm{~B}(0-5 \mathrm{~min})$, 5-95\% B (5-30 min). The injection volume was $10 \mu \mathrm{l}$, and the flow rate was $0.6 \mathrm{ml} / \mathrm{min}$. The UV chromatogram of minecoside was acquired at $330 \mathrm{~nm}$ and integrated. Purity of minecoside was $90.4 \pm 0.4 \%$. The chemical structure of MIN is shown in Fig. 1A. As preliminary experiments with minecoside (MIN) had been performed by the authors, identical conditions were adhered to throughout the study (15).

Materials. Antibodies against phospho-STAT3 (1:1,000; rabbit, monoclonal; cat. no. 9145), STAT3 (1:1,000; rabbit, monoclonal; cat. no. 12640), p-JAK1 (1:1,000; rabbit, polyclonal; cat. no. 3331), p-JAK2 (1:1,000; rabbit, monoclonal; cat. no. 8082), p-Src (1:1,000; rabbit, polyclonal; cat. no. 2101), Src (1:1,000; rabbit, polyclonal; cat. no. 2108), SHP-1 (1:1,000; rabbit, monoclonal; cat. no. 3759), cleaved PARP $(1: 1,000$; rabbit, monoclonal; cat. no. 5625), cleaved caspase-9 (1:1,000; rabbit, monoclonal; cat. no. 7237), cleaved caspase-3 (1:1,000; rabbit, polyclonal; cat. no. 9661), Bcl-2 (1:1,000; rabbit, monoclonal; cat. no. 3498), $\beta$-actin (1:1,000; rabbit, monoclonal; cat. no. 4970) and anti-rabbit $\operatorname{IgG}(1: 5,000$; rabbit, polyclonal; cat. no. 14708) were obtained from Cell Signaling Technology, Inc. CXCR4 antibody (1:10,000; rabbit, polyclonal; cat. no. ab227767) was obtained from Abcam. Antibodies to VEGF (1:1,000; rabbit, polyclonal; cat. no. sc-152), JAK1 (1:1,000; rabbit, polyclonal; cat. no. sc-277), JAK2 (1:1,000; rabbit, polyclonal; cat. no. sc-278), Bcl-xL (1:1,000; mouse, monoclonal; cat. no. sc-8392), Cyclin D1 (1:1,000; rabbit, polyclonal; cat. no. sc-718) and goat anti-mouse $\operatorname{IgG}(1: 5,000$; mouse, monoclonal; cat. no. 2355) were purchased from Santa Cruz Biotechnology, Inc. RIPA buffers were purchased from Cell Signaling Technology, Inc. DMEM, fetal bovine serum (FBS), and antibiotic-antimycotic mixture were purchased from Gibco BRL; Thermo Fisher Scientific, Inc. The DIG gel shift kit for EMSA was purchased from Roche Diagnostics.

Cell culture. MDA-MB-231 cells were obtained from the American Type Culture Collection and cultured in DMEM supplemented with $10 \%$ FBS and $1 \%$ antibiotics at $37^{\circ} \mathrm{C}$ in a humidified incubator with $5 \% \mathrm{CO}_{2}$. For the western blot assay, MDA-MB-231 cells were treated with the indicated concentrations of $\operatorname{MIN}(0,12.5,25$, and $50 \mu \mathrm{M})$ for $24 \mathrm{~h}$ or at the indicated times $(0,6,12$, and $24 \mathrm{~h})$ at $50 \mu \mathrm{M}$ concentration.

Cell viability assay by Cell Counting Kit- 8 (CCK-8). To determine the optimal concentration of MIN capable of inhibiting STAT3 activity, MDA-MB-231 cells $\left(5 \times 10^{4}\right.$ cells/well) were seeded into 96-well plates. MIN was added at various concentrations $(0-100 \mu \mathrm{M})$ at $37^{\circ} \mathrm{C}$ for $24 \mathrm{~h}$. Subsequently, $10 \mu \mathrm{l}$ CCK-8 solution was added to each well, and the cells were incubated at $37^{\circ} \mathrm{C}$ for $2 \mathrm{~h}$. The cell viability was determined by measuring the absorbance at $490 \mathrm{~nm}$ using a GloMax ${ }^{\circledR}$ Explorer Multimode Microplate Reader (Promega Corporation).

Western blot assay. As described in a previous study (17), whole-cell extracts were lysed with RIPA buffer and then the extracted proteins were separated by $10 \%$ SDS-PAGE and electrotransferred to PVDF membranes. The membranes were immunoblotted with the aforementioned primary and secondary antibodies.

Electrophoretic mobility shift assay (EMSA). As previously described (17), binding activity of STAT3 to consensus oligonucleotides was measured with extracting nuclear proteins from MIN-treated MDA-MB-231 cells using non-radioactive EMSA assay (DIG Gel Shift Kit; Roche Diagnostics). Oligonucleotide probes labeled with DIG containing consensus binding sites for STAT3 (5-CTTCATTTCCCGTAAATC CCTAAAGCT-3 and 5-AGCTTTAGGGATTTACGGGAA ATGA-3) were used.

Immunofluorescence assay. As described in a previous study (15), immunofluorescence assay was performed to check STAT3 nuclear translocation. The cells were blocked with 5\% BSA for $1 \mathrm{~h}$ and then incubated with the anti-STAT3 antibody at room temperature for $1 \mathrm{~h}$. After being washed with PBS, the slides were incubated with the secondary antibody Alexa Flour 488 for $30 \mathrm{~min}$ and counterstained for nuclei with Hoechst-33342 at $37^{\circ} \mathrm{C}$ for $10 \mathrm{~min}$. The fluorescence image was measured under x200 magnification using a fluorescence microscope (Nikon ECLIPSE Ti-U; Nikon Corporation).

TUNEL assay. Detection of DNA fragments in situ using terminal deoxyribonucleotidyl transferase-mediated dUTP-digoxigenin nick end labeling (TUNEL) assay kits (Roche Diagnostics) was applied to investigate active cell death. Briefly, cells were treated with $50 \mu \mathrm{M}$ MIN for $24 \mathrm{~h}$, then washed with PBS. The cells were fixed with freshly prepared $4 \%$ paraformaldehyde for $1 \mathrm{~h}$ at room temperature and treated with 0.2\% Triton X-100 solution for 2 min on ice. Intracellular DNA fragments were then labeled by exposing the cells to TUNEL reaction mixture for $1 \mathrm{~h}$ at $37^{\circ} \mathrm{C}$ in a humidified atmosphere, with protection from light. The cells were washed with PBS twice, then transferred to slides and analyzed under a fluorescence microscope (Nikon ECLIPSE Ti-U; Nikon Corporation).

Statistical analysis. Experimental data are presented as the mean \pm SEM obtained from three independent experiments. Statistical significance was assessed by one-way analysis of variance (ANOVA) followed by Tukey's honest significant difference test or Students' t-test using graph pad prism 6 software package (GraphPad Software Inc.) and ImageJ (imagej. nih.gov/ij). The statistics of the cell viability assay and western blot were determined by triplicate experiments using one-way ANOVA with Tukey's post hoc test. The statistical analysis of STAT3 translocation was determined by Students' t-test. P $<0.05$ was considered to indicate a statistically significant difference. 


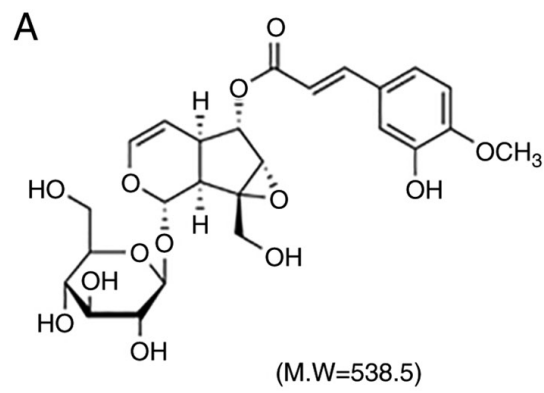

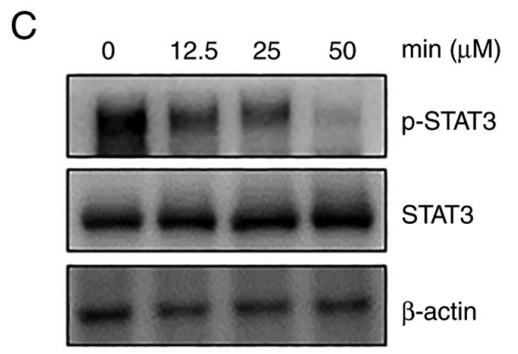

D

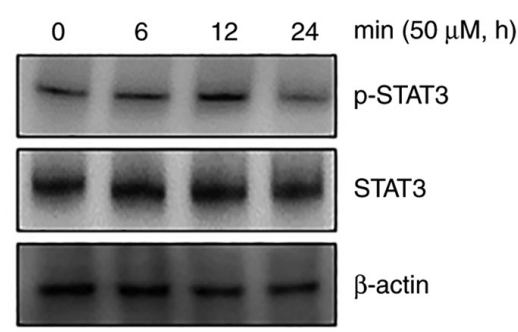

B
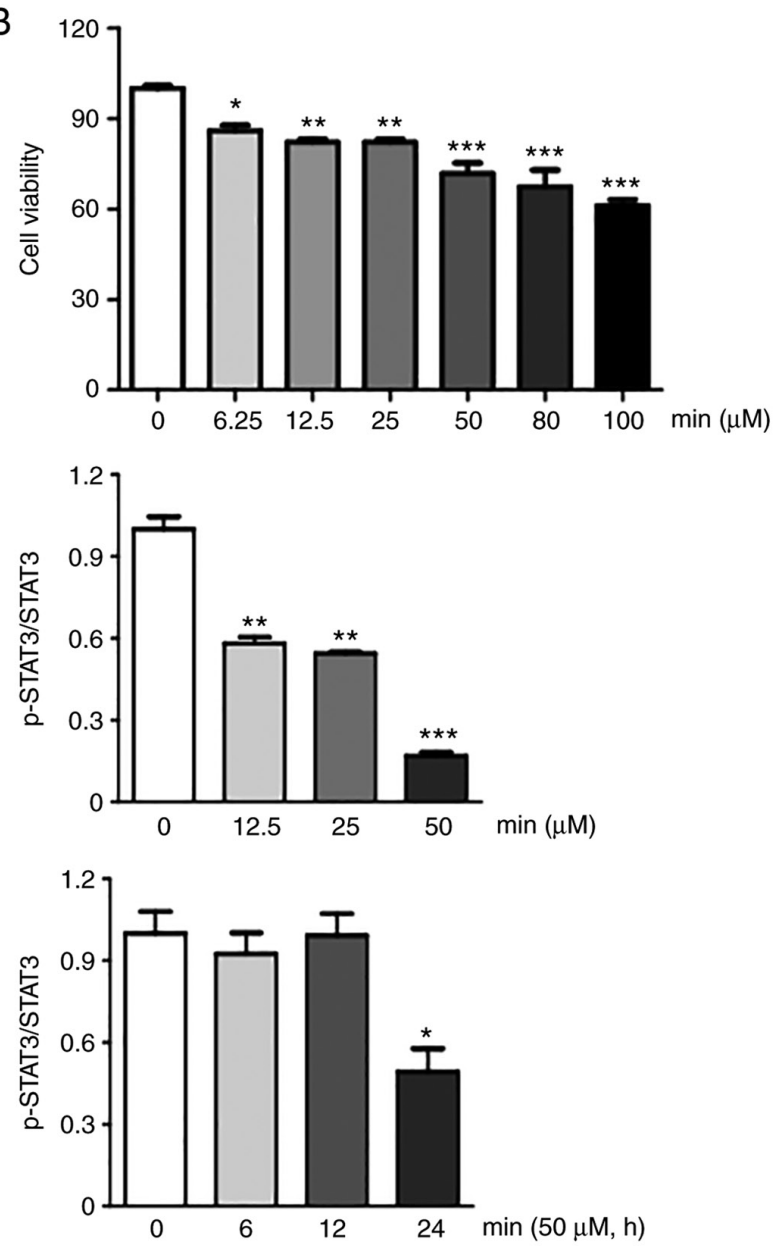

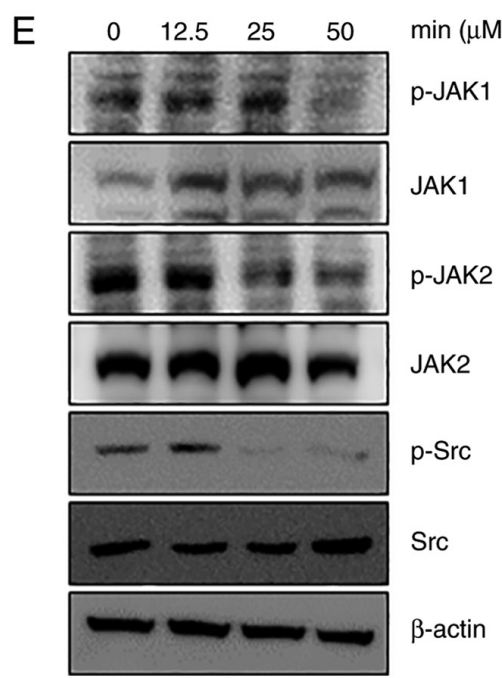

Figure 1. MIN downregulates constitutive activation of STAT3 in MDA-MB-231 cells. (A) Chemical structure of minecoside (MIN) (B) MDA-MB-231 cells $\left(1 \times 10^{4}\right.$ cells $\left./ \mathrm{ml}\right)$ were treated with the indicated concentrations of MIN. The cell viability was assessed by CCK- 8 assays and the results are expressed as percentage of surviving cells over control cells. (C) MIN inhibits STAT3 phosphorylation in a dose-dependent manner. MDA-MB-231 cells were treated with the indicated concentrations of MIN for $24 \mathrm{~h}$. Subsequently, western blotting was performed as described in Materials and methods. (D) MIN inhibits STAT3 phosphorylation in a time-dependent manner. Western blotting was performed after MDA-MB-231 cells were treated with $50 \mu \mathrm{M}$ MIN for the indicated times $(0,6,12$ and $24 \mathrm{~h})$. (E) MIN suppresses p-JAK1, p-JAK2 and p-Src expression in a dose-dependent manner. After MDA-MB-231 cells were treated with the indicated concentrations of MIN for $24 \mathrm{~h}$, the protein expression of p-JAK1, p-JAK2 and p-Src was observed by western blot analysis. Data indicate mean \pm SEM of three independent experiments. ${ }^{*} \mathrm{P}<0.05,{ }^{* *} \mathrm{P}<0.01,{ }^{* * *} \mathrm{P}<0.001$ vs. control.

\section{Results}

MIN downregulates constitutive activation of STAT3 in $M D A-M B-231$ cells. Since constitutive activation of STAT3 is found in numerous cancer types, including $>40 \%$ of primary breast tumors (4), and a recent study has reported anti-cancer effects of MIN in breast cancer (15), whether MIN affects STAT3 activation was examined in the present study. To 
A

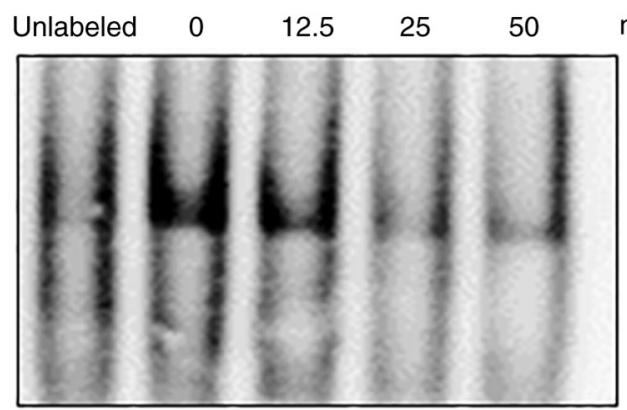

STAT3
B

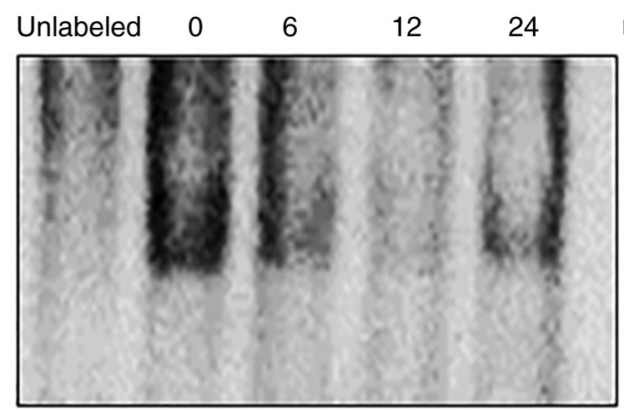

$\min (50 \mu \mathrm{M}, \mathrm{h})$

STAT3
C
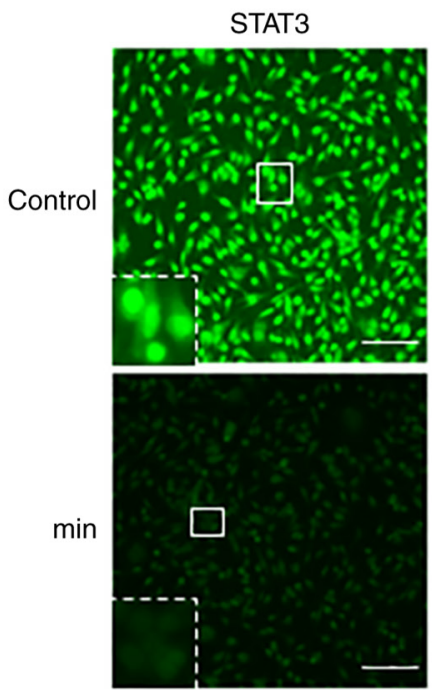

Hoechst 33342
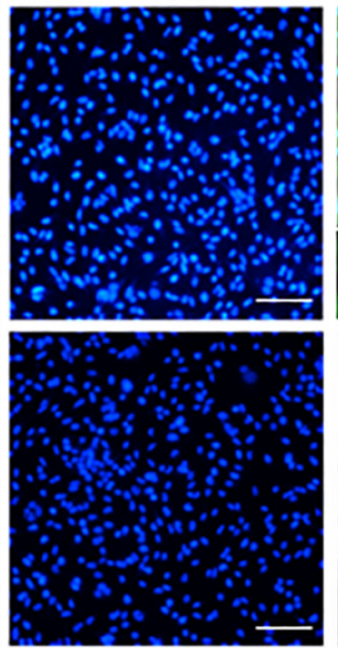
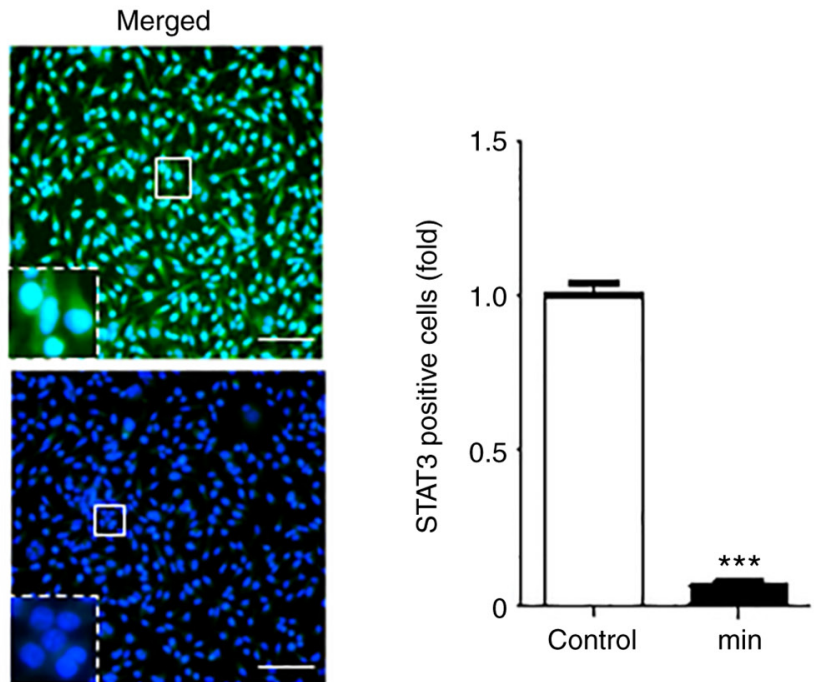

Figure 2. MIN suppresses DNA binding of STAT3 in MDA-MB-231 cells. (A) MIN suppresses STAT3-DNA binding activity in a dose-dependent manner. MDA-MB-231 cells were treated with the indicated concentrations of MIN for $24 \mathrm{~h}$ and STAT3-DNA binding activity was measured by EMSA. (B) MDA-MB-231 cells were treated with $50 \mu \mathrm{M}$ MIN for the indicated times and analyzed for nuclear STAT3 levels by EMSA. (C) MIN inhibits nuclear translocation of STAT3 in MDA-MB-231 cells. After treatment with MDA-MB-231 cells with $50 \mu \mathrm{M}$ MIN for $24 \mathrm{~h}$, nuclear translocation of STAT3 was measured by immunofluorescence. Scale bars: $100 \mu \mathrm{m}$ (magnification, x200). The third panels show the merged images of the first and second panels. The fold of STAT3 positive cells with fluorescence was quantified using ImageJ. ${ }^{* * *} \mathrm{P}<0.001$ vs. control.

determine the optimal concentration of MIN, the cell viability at the indicated concentrations of $\operatorname{MIN}(0,6.25,12.5,25,50$, 80 and $100 \mu \mathrm{M}$ ) were evaluated. Based on the results, $50 \mu \mathrm{M}$ of MIN was selected as an optimal concentration and used throughout the study (Fig. 1B). As shown in Fig. 1C and D, MIN reduced the phosphorylation of STAT3 in a dose- and time-dependent manner in MDA-MB-231 cells. The reduction ratio of phosphorylated STAT3 to STAT3 was from 15 to $55 \%$.

MIN downregulates upstream signaling pathway responsible for STAT3 activation. Janus kinases 1/2 (JAK1/2) and Src kinase were reported to contribute to STAT3 activation and are involved in cancer cell growth (18). Thus, whether MIN could inhibit the upstream signaling molecules that activate STAT3 was examined. The results showed that MIN suppressed the constitutive activation of JAK1, JAK2, and Src kinase at $50 \mu \mathrm{M}$ in MDA-MB-231 cells (Fig. 1E).

MIN suppresses DNA binding of STAT3 in MDA-MB-23I cells. Based on the fact that the phosphorylation of STAT3 regulates gene transcription through dimerization, nuclear translocation, and DNA binding (19), whether MIN can regulate the DNA binding activity of STAT3 was investigated.
After cells were treated with the indicated concentrations of MIN for $24 \mathrm{~h}$ or with $50 \mu \mathrm{M}$ of MIN for different time periods, EMSA was performed to examine the DNA binding activity of STAT3. The results showed that MIN suppressed STAT3-DNA binding in a dose- and time-dependent manner (Fig. 2A and B).

To check whether MIN inhibits STAT3-DNA binding by blocking STAT3 nuclear translocation, an immunofluorescence assay was performed. As shown in Fig. $2 \mathrm{C}, 50 \mu \mathrm{M}$ MIN suppressed the translocation of STAT3 into the nucleus as compared to the control groups.

MIN induces the expression of SHP-1. Previous findings showed that, several PTPs can modulate the STAT3 signaling pathway in various cancer cells (20). Therefore, whether the regulation of STAT3 activity by MIN was due to PTP induction was examined. Several studies reported that loss of SHP-1 in cancer cells improves STAT3 signaling $(12,21)$. In addition, SHP-1 was proposed as a promising drug target in the development of STAT3 inhibitors because it can dephosphorylate JAKs (22) as well as STAT3 (11). Thus, whether MIN modulated SHP-1 expression in MDA-MB-231 cells was examined. As shown in Fig. 3A, MIN treatment increased SHP-1 expression in a concentration-dependent manner. 
A

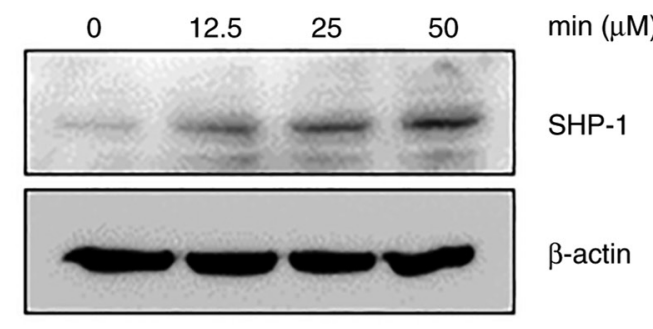

B

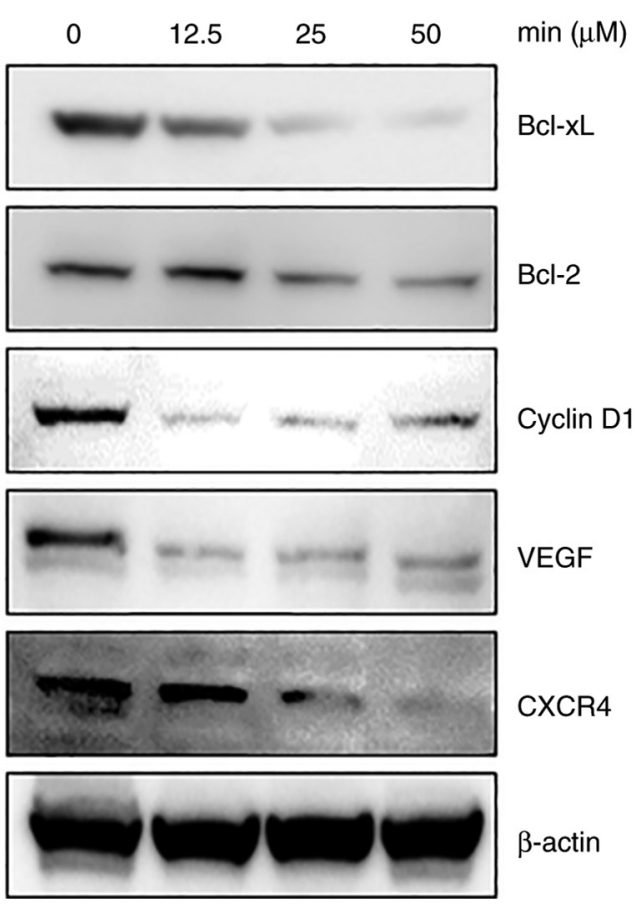

C
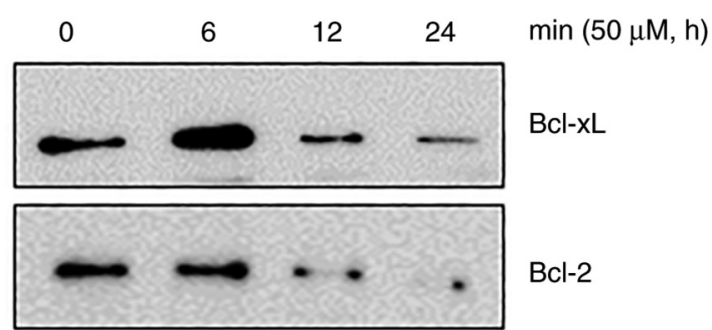

$\mathrm{Bcl}-2$

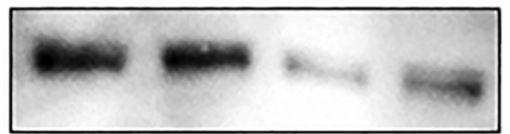

Cyclin D1
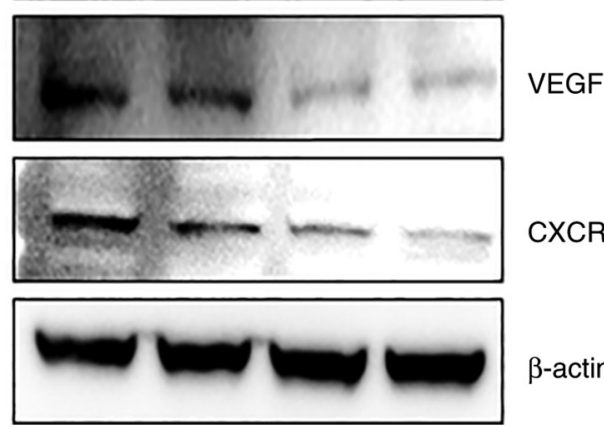

CXCR4

$\beta$-actin

Figure 3. MIN inhibits the expression of various genes involved in growth, angiogenesis, invasion, and metastasis. (A) MIN induces the expression of SHP-1 protein in MDA-MB-231 cells. After MDA-MB-231 cells were treated with indicated concentrations of MIN for $24 \mathrm{~h}$, whole cell extracts were prepared and the protein expression of SHP-1 was observed by western blot analysis. (B and C) MIN suppresses various gene products involved in survival, apoptosis, and invasion.

MIN inhibits the expression of various genes involved in growth, angiogenesis, invasion, and metastasis. STAT3 activation induces the expression of various genes involved in cancer cell survival and proliferation (9). The anti-apoptotic proteins Bcl-2 (23), Bcl-xL (24), invasive protein CXCR4 (25), angiogenic protein VEGF (26), and cell cycle control protein cyclin D1 (27) are known to be induced upon STAT3 activation. Thus, whether MIN affects the expression of these genes by regulating STAT3 activity was investigated. As shown in Fig. 3B and C, MIN downregulated the expression of Bcl-xL, Bcl-2, cyclin D1, VEGF, and CXCR4 in a dose- and time-dependent manner.

MIN induces caspase-dependent apoptosis in MDA-MB-231 cells. To further examine the apoptotic progression induced by MIN, major proteins involved in the caspase pathway were examined using western blot analysis. The results showed that MIN upregulated the expression of cleaved forms of caspase-9, caspase-3, and PARP in a dose- and time-dependent manner (Fig. 4A and B). To confirm MIN-induced apoptosis, a TUNEL assay, which is a commonly used method for identifying apoptosis, was performed. The TUNEL analysis revealed that MIN increased the number of apoptotic cells (Fig. 4C). Taken together, these results strongly suggested that MIN induced caspase-dependent apoptosis in MDA-MB-231 cells.

\section{Discussion}

Although the specific role of STAT3 in the onset and progression of breast cancer is not fully defined, the activation of IL-6/JAK2/STAT3 pathways is well known to promote carcinogenesis $(4,28)$. In particular, breast cancer is associated with inflammatory conditions with high levels of growth factors and cytokines (6). For example, inflammatory cytokines, such as IL-6, are known to promote cancer cell growth, invasion, migration, metastasis, angiogenesis, and drug resistance through the JAK/STAT3 pathway $(23,26,29)$. Based on the aforementioned studies, we investigated whether MIN promoted apoptosis by inhibiting constitutive STAT3 activation in breast cancer cells.

The results showed that, MIN suppressed constitutive STAT3 activation in MDA-MB-231 cells. The phosphorylation of STAT3 at Tyr705, an important step in STAT3 activation, was inhibited by MIN in a dose- and time-dependent manner. STAT3 phosphorylation is mediated through the activation of non-receptor protein tyrosine kinases such as JAK1 and JAK2. Src kinase is also known to play 
A

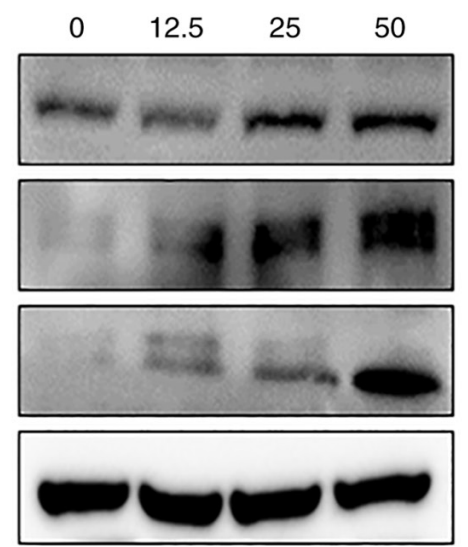

C
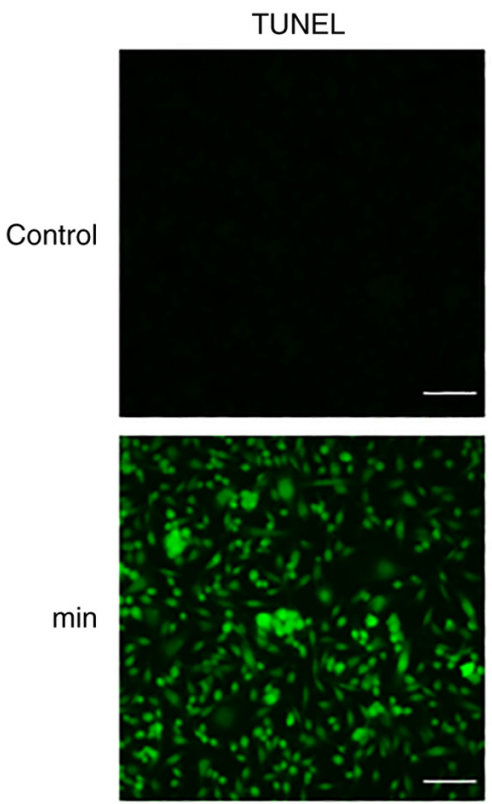

$\min (\mu \mathrm{M})$

Cleaved PARP

Cleaved caspase-9

Cleaved caspase-3

$\beta$-actin
B
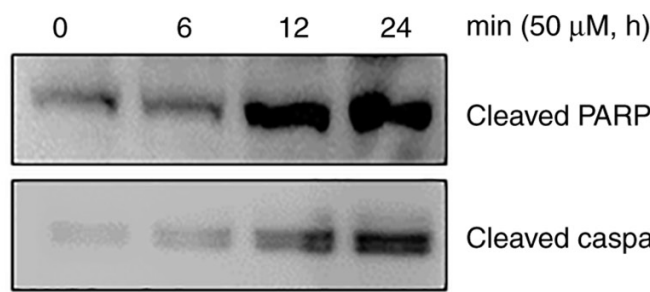

Cleaved caspase-9

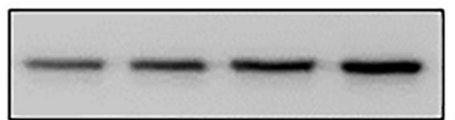

Cleaved caspase-3

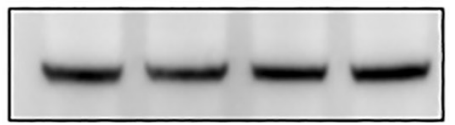

$\beta$-actin
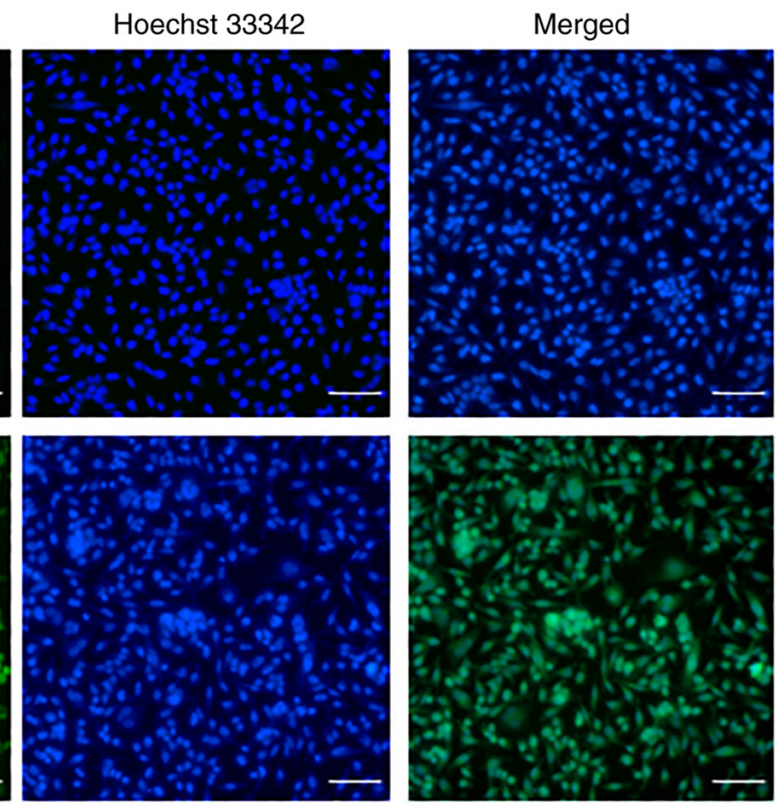

Figure 4. MIN induces caspase-dependent apoptosis in MDA-MB-231 cells. (A and B) MIN induces cleavage of caspase-9, -3, and PARP. Whole cell extracts were subjected to western blotting against a cleaved form of caspase-9, caspase-3 and PARP. (C) MDA-MB-231 cells were treated with $50 \mu \mathrm{M}$ MIN for $24 \mathrm{~h}$, fixed with $4 \%$ paraformaldehyde, permeabilized with $0.2 \%$ Triton X-100, and stained with labeled TUNEL reagent. The third panels show the merged images of the first and second panels. Scale bars: $100 \mu \mathrm{m}$ (magnification, $\mathrm{x} 200$ ).

an important role in the phosphorylation of STAT3 $(30,31)$ Indeed, although upstream kinases are important in tumorigenesis, STAT3, which is considered a key transcription factor for gene expression involved in malignancy, was the primary focus of the present study. The results demonstrated that MIN inhibited JAK1, JAK2, and Src kinase activation, suggesting that MIN suppresses STAT3 phosphorylation via the inactivation of upstream kinases. Since the phosphorylation of STAT3 regulates gene transcription through dimerization, nuclear translocation, and DNA binding (19), whether MIN can affect STAT3 translocation and DNA binding activity was investigated. The results revealed that MIN suppressed STAT3-DNA binding as well as the nuclear translocation of STAT3.

Several PTPs modulate the STAT3 signaling pathway in various cancer cells (20). In particular, the $\mathrm{SH} 2$ domain-containing phosphatase-1 (SHP-1) possesses a tumor-suppressive potential by virtue of negatively regulating STAT3 signaling (12); it is considered an antagonist of tyrosine kinases related to tumor growth and anti-apoptosis (32). SHP-1 expression is known to be reduced or abolished in estrogen receptor-negative breast cancer cell lines. In the present study, whether the inhibitory effect of MIN on STAT3 activation was related to SHP-1 expression was examined. The results showed that, MIN treatment increased SHP-1 expression in a concentration-dependent manner. The increase in SHP-1 expression by MIN was confirmed via the association with the inhibition of constitutive STAT3 activation in subsequent experiments. Besides SHP-1, the involvement of other PTPs in the regulation of STAT3 by MIN should be investigated in future studies.

STAT3 activation is observed in various malignant tumors, including lung, breast, liver, colon, prostate, stomach, pancreas, kidney, and brain cancers (33). This is because STAT3 activation upregulates a variety of cellular signaling required for cell survival (Bcl-xL, Bcl-2, c-myc, Mcl-1, and survivin), proliferation (cyclin D1), invasion (MMP-9, CXCR4, Rho, and Rac), and angiogenesis (VEGF) $(23,25,28,33)$. Findings of the present study showed that MIN also downregulated STAT3-mediated protein 
expression such as cyclin D1, Bcl-2, Bcl-xL, CXCR4, and VEGF. In a previous study, the inhibitory effect of MIN on CXCR4 expression was found to be mediated via the blockade of transcription factor NF- $\kappa \mathrm{B}$ (15). A gene that contains only $\mathrm{NF}-\kappa \mathrm{B}$ binding sites may be responsive to $\mathrm{NF}-\kappa \mathrm{B}$, but not STAT3. However, a gene that contains both $\mathrm{NF}-\kappa \mathrm{B}$ and STAT3 binding sites may be regulated by both in a cooperative manner (34). Thus, investigation of the key role of MIN on crosstalk between STAT3 and NF- $\kappa \mathrm{B}$ is imperative.

Since MIN inhibited the expression of proteins related to proliferation (cyclin D1), and cell survival (Bcl-2, Bcl-xL), whether MIN affected apoptotic progression was examined. The results showed that MIN upregulated caspase-dependent apoptosis in MDA-MB-231 cells. A major limitation of the present study was that the inhibitory effect of MIN was not examined in other cancer cells or in an animal model.

In conclusion, the results of the present study provide evidence that MIN exerts anticancer activity via inhibition of the JAK/STAT3 signaling pathway, especially in breast cancer cells. Further studies using animal models are required to determine the potential of this molecule as an anticancer drug.

\section{Acknowledgements}

Not applicable.

\section{Funding}

This research was supported by a grant from the Korea Health Technology R\&D Project through the Korea Health Industry Development Institute (KHIDI), funded by the Ministry of Health \& Welfare, Republic of Korea (HF20C0038).

\section{Availability of data and materials}

All data generated or analyzed during the present study are included in this published article.

\section{Authors' contributions}

BP and BK conceived the study. BK developed the methodology, and obtained and validated the data. BK performed the experiments. BP and KL analyzed and interpreted the data. $\mathrm{BK}$ and $\mathrm{BP}$ prepared the original draft. BP and KL revised the draft. BK and BP confirm the authenticity of all the raw data. All authors read and approved the final manuscript.

\section{Ethics approval and consent to participate}

Not applicable.

\section{Patient consent for publication}

Not applicable.

\section{Competing interests}

The authors declare that they have no competing interests.

\section{References}

1. DeSantis C, Siegel R, Bandi P and Jemal A: Breast cancer statistics, 2011. CA Cancer J Clin 61: 409-418, 2011.

2. Foulkes WD, Smith IE and Reis-Filho JS: Triple-negative breast cancer. N Engl J Med 363: 1938-1948, 2010.

3. Dean M, Fojo T and Bates S: Tumour stem cells and drug resistance. Nat Rev Cancer 5: 275-284, 2005.

4. Banerjee $\mathrm{K}$ and Resat $\mathrm{H}$ : Constitutive activation of STAT3 in breast cancer cells: A review. Int J Cancer 138: 2570-2578, 2016.

5. Laudisi F, Cherubini F, Monteleone G and Stolfi C: STAT3 interactors as potential therapeutic targets for cancer treatment. Int J Mol Sci 19: 1787, 2018.

6. Garbers C, Aparicio-Siegmund S and Rose-John S: The IL-6/gp130/STAT3 signaling axis: Recent advances towards specific inhibition. Curr Opin Immunol 34: 75-82, 2015.

7. Becker C, Fantini MC, Schramm C, Lehr HA, Wirtz S, Nikolaev A, Burg J, Strand S, Kiesslich R, Huber S, et al: TGF-beta suppresses tumor progression in colon cancer by inhibition of IL-6 trans-signaling. Immunity 21: 491-501, 2004.

8. Kundu J, Choi BY, Jeong CH, Kundu JK and Chun KS: Thymoquinone induces apoptosis in human colon cancer HCT116 cells through inactivation of STAT3 by blocking JAK2and Src-mediated phosphorylation of EGF receptor tyrosine kinase. Oncol Rep 32: 821-828, 2014.

9. Wang X, Crowe PJ, Goldstein D and Yang JL: STAT3 inhibition, a novel approach to enhancing targeted therapy in human cancers (Review). Int J Oncol 41: 1181-1191, 2012.

10. Gu F, Dubé N, Kim JW, Cheng A, Ibarra-Sanchez Mde J, Tremblay ML and Boisclair YR: Protein tyrosine phosphatase $1 \mathrm{~B}$ attenuates growth hormone-mediated JAK2-STAT signaling. Mol Cell Biol 23: 3753-3762, 2003.

11. Liu CY, Tseng LM, Su JC, Chang KC, Chu PY, Tai WT, Shiau CW and Chen KF: Novel sorafenib analogues induce apoptosis through SHP-1 dependent STAT3 inactivation in human breast cancer cells. Breast Cancer Res 15: R63, 2013.

12. Wu C, Sun M, Liu L and Zhou GW: The function of the protein tyrosine phosphatase SHP-1 in cancer. Gene 306: 1-12, 2003.

13. Joo MK, Park JJ, Yoo HS, Lee BJ, Chun HJ, Lee SW and Bak YT: Epigenetic regulation and anti-tumorigenic effects of SH2-containing protein tyrosine phosphatase 1 (SHP1) in human gastric cancer cells. Tumour Biol 37: 4603-4612, 2016.

14. Kwak JH, Kim HJ, Lee KH, Kang SC and Zee OP: Antioxidative iridoid glycosides and phenolic compounds from Veronica peregrina. Arch Pharm Res 32: 207-213, 2009.

15. Kim B, Min YH and Park B: Minecoside modulates cell invasion via regulation of CXCR4 expression in breast and colon cancer cells. Planta Med 86: 331-337, 2020.

16. Park S, Shin H, Park Y, Choi I, Park B and Lee KY: Characterization of inhibitory constituents of NO production from Catalpa ovata using LC-MS coupled with a cell-based assay. Bioorg Chem 80: 57-63, 2018.

17. Kim B, Lee KY and Park B: Crocin suppresses constitutively active STAT3 through induction of protein tyrosine phosphatase SHP-1. J Cell Biochem 118: 3290-3298, 2017.

18. Yu H, Lee H, Herrmann A, Buettner R and Jove R: Revisiting STAT3 signalling in cancer: New and unexpected biological functions. Nat Rev Cancer 14: 736-746, 2014.

19. Masciocchi D, Gelain A, Villa S, Meneghetti F and Barlocco D: Signal transducer and activator of transcription 3 (STAT3): A promising target for anticancer therapy. Future Med Chem 3: 567-597, 2011.

20. Kim M, Morales LD, Jang IS, Cho YY and Kim DJ: Protein tyrosine phosphatases as potential regulators of STAT3 signaling. Int J Mol Sci 19: 2708, 2018.

21. Han Y, Amin HM, Franko B, Frantz C, Shi X and Lai R: Loss of SHP1 enhances JAK3/STAT3 signaling and decreases proteosome degradation of JAK3 and NPM-ALK in $\mathrm{ALK}^{+}$anaplastic large-cell lymphoma. Blood 108: 2796-2803, 2006.

22. Jiao H, Berrada K, Yang W, Tabrizi M, Platanias LC and Yi T: Direct association with and dephosphorylation of Jak2 kinase by the SH2-domain-containing protein tyrosine phosphatase SHP-1. Mol Cell Biol 16: 6985-6992, 1996.

23. Real PJ, Sierra A, De Juan A, Segovia JC, Lopez-Vega JM and Fernandez-Luna JL: Resistance to chemotherapy via Stat3-dependent overexpression of Bcl-2 in metastatic breast cancer cells. Oncogene 21: 7611-7618, 2002.

24. Wang J, Xu J and Xing G: Lycorine inhibits the growth and metastasis of breast cancer through the blockage of STAT3 signaling pathway. Acta Biochim Biophys Sin (Shanghai) 49: 771-779, 2017. 
25. Liu X, Xiao Q, Bai X, Yu Z, Sun M, Zhao H, Mi X, Wang E, Yao W, Jin F, et al: Activation of STAT3 is involved in malignancy mediated by CXCL12-CXCR4 signaling in human breast cancer. Oncol Rep 32: 2760-2768, 2014.

26. Niu G, Wright KL, Huang M, Song L, Haura E, Turkson J, Zhang S, Wang T, Sinibaldi D, Coppola D, et al: Constitutive Stat 3 activity up-regulates VEGF expression and tumor angiogenesis. Oncogene 21: 2000-2008, 2002.

27. Johnston PA and Grandis JR: STAT3 signaling: Anticancer strategies and challenges. Mol Interv 11: 18-26, 2011.

28. Burke WM, Jin X, Lin HJ, Huang M, Liu R, Reynolds RK and Lin J: Inhibition of constitutively active Stat 3 suppresses growth of human ovarian and breast cancer cells. Oncogene 20: 7925-7934, 2001.

29. Wang T, Niu G, Kortylewski M, Burdelya L, Shain K, Zhang S Bhattacharya R, Gabrilovich D, Heller R, Coppola D, et al: Regulation of the innate and adaptive immune responses by Stat-3 signaling in tumor cells. Nat Med 10: 48-54, 2004.

30. Ihle JN: STATs: Signal transducers and activators of transcription. Cell 84: 331-334, 1996.
31. Schreiner SJ, Schiavone AP and Smithgall TE: Activation of STAT3 by the Src family kinase Hck requires a functional SH3 domain. J Biol Chem 277: 45680-45687, 2002.

32. Huang TT, Su JC, Liu CY, Shiau CW and Chen KF: Alteration of SHP-1/p-STAT3 signaling: A potential target for anticancer therapy. Int J Mol Sci 18: 1234, 2017.

33. Santoni M, Massari F, Del Re M, Ciccarese C, Piva F, Principato G, Montironi R, Santini D, Danesi R, Tortora G and Cascinu S: Investigational therapies targeting signal transducer and activator of transcription 3 for the treatment of cancer. Expert Opin Investig Drugs 24: 809-824, 2015.

34. Grivennikov SI and Karin M: Dangerous liaisons: STAT3 and NF-kappaB collaboration and crosstalk in cancer. Cytokine Growth Factor Rev 21: 11-19, 2010.

This work is licensed under a Creative Commons Attribution-NonCommercial-NoDerivatives 4.0 International (CC BY-NC-ND 4.0) License. 